\title{
Compositional variations along the route of Chang'e-3 Yutu rover revealed by the lunar penetrating radar
}

\author{
Chunyu Ding ${ }^{1,2}$, Zhiyong Xiao ${ }^{1,3,4^{*}}$ (D), Yan Su, ${ }^{2,5}$, Jiannan Zhao ${ }^{6}$ and Jun Cui ${ }^{1,2,4}$
}

\begin{abstract}
Using the high-frequency lunar penetrating radar data obtained by the Chang'e-3 mission, we apply the frequencyshift method to calculate the decay rate of the electromagnetic wave in the regolith-like ejecta deposits of the Ziwei crater. The radar data are divided into segments according to the navigation points along the traverse route of the Yutu rover. For each segment, we calculate the bulk loss tangent of materials within the top $\sim 50 \mathrm{~ns}$ of the radar data based on the frequency decreasing rate of the electromagnetic wave. The loss tangent varies from 0.011-0.017 along the route of Yutu, and it is within the range of the measured loss tangent of Apollo regolith samples. Using the empirical relationship between loss tangent and $\mathrm{TiO}_{2}+\mathrm{FeO}$ content derived from the Apollo lunar samples, we estimate the $\mathrm{TiO}_{2}+\mathrm{FeO}$ content for the bulk regolith along the route of Yutu, which is $~ 23-30$ wt.\%. This value is comparable with that estimated using both orbital reflectance spectral data and in situ observation made by the Yutu rover. The loss tangent derived along the route of Yutu is larger than the average value of returned lunar samples, which is mainly caused by the larger content of $\mathrm{TiO}_{2}+\mathrm{FeO}$ at the landing site compared to the global average. Variations of the $\mathrm{TiO}_{2}+\mathrm{FeO}$ content along the route of Yutu are mainly due to the excavation of the Ziwei crater. The $\mathrm{TiO}_{2}+\mathrm{FeO}$ content map derived by the radar has a much higher spatial resolution compared to orbital observation, testifying the feasibility of this technique for regional geology study.
\end{abstract}

Keywords: Moon, Regolith, Ground penetrating radar, Loss tangent, Chang'e-3

\section{Introduction}

Most of our knowledge about the composition of the Moon is derived from orbital observations, since the number of both returned lunar samples and collected lunar meteorites is still rather limited. Orbital observations using spectrometers that work at various wavelengths have perfect spatial coverage, but they are usually restricted in the spatial resolution. On the other hand, highly heterogeneous materials exist at any places of the Moon due to intense material transportation, mixing, and metamorphism by impact cratering (Huang

\footnotetext{
* Correspondence: xiaozhiyong@mail.sysu.edu.cn

'Planetary Environmental and Astrobiological Research Laboratory, School of Atmospheric Sciences, Sun Yat-sen University, Zhuhai, China

${ }^{3}$ State Key Laboratory of Lunar and Planetary Sciences, Space Science Institute, Macau University of Science and Technology, Macau, China Full list of author information is available at the end of the article
}

et al. 2017). Therefore, there is a gap in the spatial resolution of interpreted compositions using orbital and laboratory measurements, and caution should be used when directly comparing the compositional data obtained from orbit and those measured for samples at laboratories. For example, reflectance spectra obtained from orbit (normally $>10 \mathrm{~m} /$ pixel) are usually compared with those measured at laboratories for typical minerals and lunar samples to determine the possible compositions (e.g., Pieters et al. 2000), but the two measurements have a scale difference of at least an order of magnitude. Recently, Wu and Hapke (2018) analyzed the visible-near-infrared spectrometer data obtained on the lunar surface by the Chang'e-3 mission (i.e., CE-3), and heterogeneous reflectance spectra were observed both at the surface and in the near-subsurface materials.

\section{Springer Open}

( ) The Author(s). 2020 Open Access This article is licensed under a Creative Commons Attribution 4.0 International License, which permits use, sharing, adaptation, distribution and reproduction in any medium or format, as long as you give appropriate credit to the original author(s) and the source, provide a link to the Creative Commons licence, and indicate if changes were made. The images or other third party material in this article are included in the article's Creative Commons licence, unless indicated otherwise in a credit line to the material. If material is not included in the article's Creative Commons licence and your intended use is not permitted by statutory regulation or exceeds the permitted use, you will need to obtain permission directly from the copyright holder. To view a copy of this licence, visit http://creativecommons.org/licenses/by/4.0/. 
Lunar penetrating radar (i.e., LPR) can also be used to deduce the composition of surface materials, especially the content of $\mathrm{TiO}_{2}+\mathrm{FeO}$ (Schaber et al. 1975). The rate of energy decay of electromagnetic waves in lunar regolith is mainly controlled by the content of $\mathrm{TiO}_{2}+$ FeO (Strangway et al. 1977), so that the loss tangent of lunar regolith derived from the energy decay rate of electromagnetic waves can be used to estimate the amount of $\mathrm{TiO}_{2}+\mathrm{FeO}$ (Campbell et al. 1997). Pommerol et al. (2010) noticed that radar echoes returned from high-Ti regions on the Moon were low, which were detected by the lunar radar sounder onboard the Selenological and Engineering Explorer Kaguya mission. Applying this method, Campbell et al. (1997) estimated the bulk composition of regolith in the lunar mare using Earth-based radar data. The LPR onboard the Yutu rover of the CE-3 mission obtained the first in situ radar profile on the surface of the Moon $(\mathrm{Su}$ et al. 2014). Compared to the radar data obtained by Earthbased telescopes (e.g., Campbell et al. 1997), the LPR data obtained by CE-3 have a much shorter wavelength ("Data and method" section) and higher spatial resolution. This work utilizes the CE-3 LPR data to derive the composition along the route of Yutu, which will fill the observation gap in terms of the spatial resolution between the orbital (e.g., Zhao et al. 2014) and in situ measurements (e.g., Wu and Hapke, 2018). Furthermore, instead of probing only the top-most materials, the LPRderived contents of $\mathrm{TiO}_{2}+\mathrm{FeO}$ represent the average value of materials within the radar detection ranges.

We introduce the data obtained by the CE-3 highfrequency LPR in the "Data" section, the frequency-shift technique used to estimate the loss tangent of lunar materials and the method used to derive the $\mathrm{TiO}_{2}+\mathrm{FeO}$ content are introduced in the "Estimate of loss and tangent". The derived loss tangent values and the $\mathrm{TiO}_{2}+$ $\mathrm{FeO}$ contents are discussed in the "Estimate of loss and tangent" and "Bulk $\mathrm{TiO}_{2}+\mathrm{FeO}$ content" sections, respectively, and the reliability of these results is verified in the "Reliability of the loss tangent estimated" and "Reliability of the $\mathrm{TiO}_{2}+\mathrm{FeO}$ contents estimated". Indications of the results are discussed in "Indications to regional geology" section.

\section{Data and method}

\section{Data}

We use the CE-3 high-frequency LPR data in this study. The LPR system onboard the Yutu rover consists of two channels that have center frequencies of 60 and 500 $\mathrm{MHz}$, respectively (Fang et al. 2014; Su et al. 2014). The transmitted pulse of the LPR was generated by a digital integrated circuit, so that the transmitted waveform was constant (Fang et al. 2014), enabling the derivation of loss tangent from the measured frequency drift rate
(Irving and Knight, 2003). The high-frequency LPR was operated with different gain values during the mission (Feng et al. 2017), and a constant gain of $0 \mathrm{~dB}$ was used from the navigation point N105 to N208 (Fig. 1). To avoid uncertainties raised by the different gain values (Feng et al. 2017), we use the radar data from N105 to N208 here, which contain $\sim 1600$ valid traces after removing the redundant data.

The radar data are processed following the routine procedure (e.g., Su et al. 2014). The Yutu rover was driven with different speeds from the navigation points N105 to N208 (Feng et al. 2017). To remedy distortions in the radargram that are caused by the different moving speeds (e.g., data sections N202-203 and N204-206 shown in Fig. 1c), we perform a trace equivalent calculation for the data based on the average rover speed (i.e., $0.055 \mathrm{~m} / \mathrm{s}$ ). Afterward, the standard processing procedure (e.g., remove direct current component and background, and band-pass filtering) is applied to obtain the final LPR radargram (Fig. 2a).

We use the top $\sim 50$ ns of the radargram for this study. There is a general consensus that this part of the radargram corresponds to the continuous ejecta deposits of the Ziwei crater (Fig. 1a, e.g., Xiao et al. 2015; Fa et al. 2015; Zhang et al. 2019), which is supported by both the geological context (Qiao et al. 2016) and the signal transition of radar echoes (Xiao et al. 2015). Data at larger depths are less clear in terms of the geological interpretation (Xiao et al. 2015; Zhang et al. 2015; Zhang et al. 2019), and the signal-to-noise ratio is lower (Xing et al. 2017). To avoid possible controversies regarding the reliability of both the data and geological interpretation at larger depths, we focus on the $\sim 50$ ns of the radargram here. Previous studies have found that materials within this depth (i.e., the continuous ejecta deposits of Ziwei) have similar properties with typical regolith materials (Lai et al. 2016; Xiao et al. 2015).

\section{Estimate of loss tangent}

We focus on the imaginary part of the relative permittivity (i.e., loss tangent) to invert the content of $\mathrm{TiO}_{2}+$ FeO. Radar permittivity consists of real and imaginary parts. For the top $\sim 50 \mathrm{~ns}$ LPR radargram, the real part of the relative permittivity has been estimated using hyperbolic reflectors in the radargram, resulting in values below 5 (Feng et al. 2017; Lai et al. 2016). However, the imagery part for the lunar surface at the CE-3 landing site was taken as a constant value of $\sim 0.014$, and this value was estimated using the empirical relationship between loss tangent and the content of $\mathrm{TiO}_{2}+-$ FeO of the surface regolith ( $\sim 27.8 \%$ on average), which was obtained by the Active Particle-induced X-ray Spectrometer (APXS) onboard the Yutu rover (Ling et al. 2015). However, the APXS observations are only for 4 


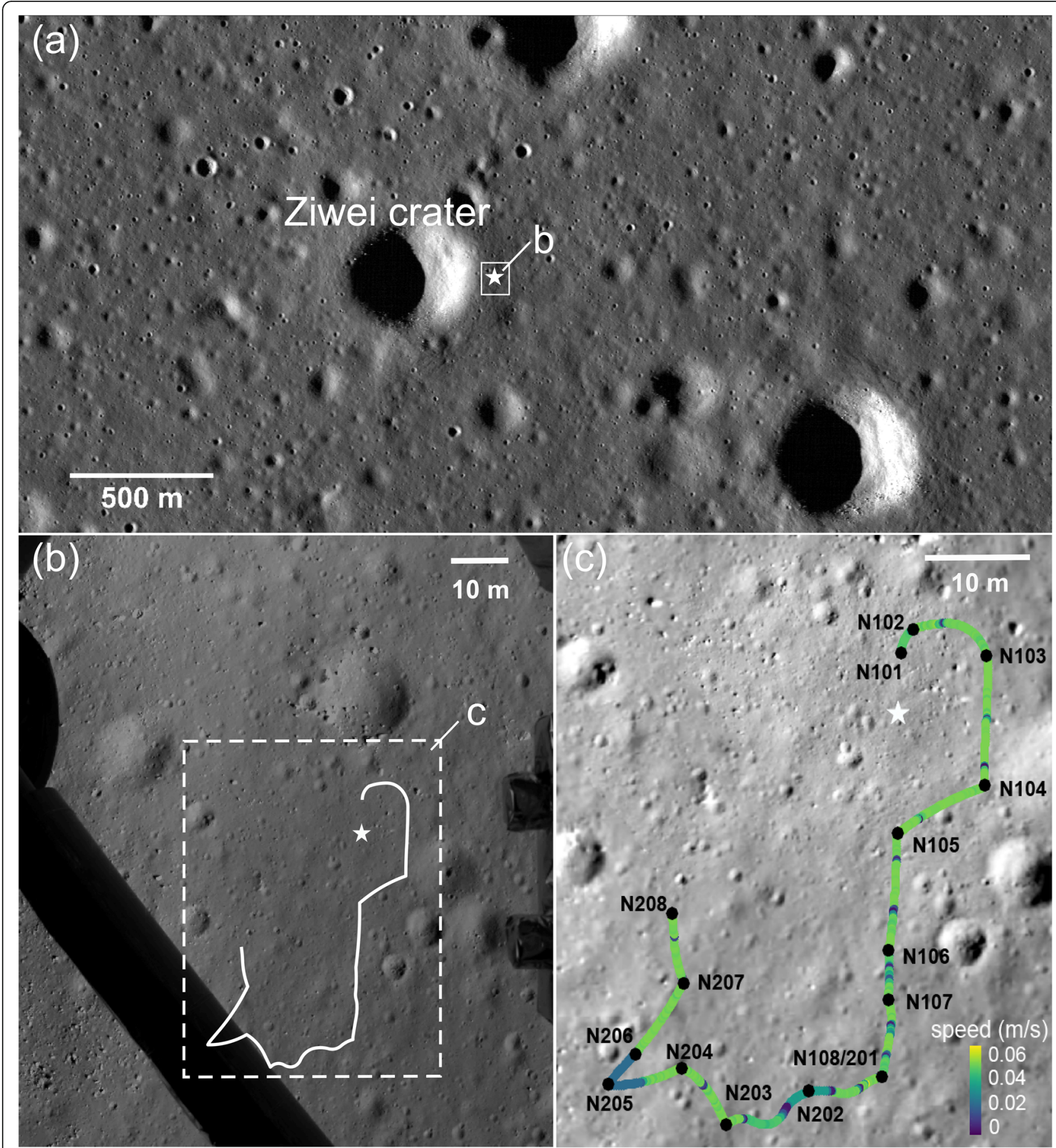

Fig. 1 The landing site of the Chang'e-3 mission and the traverse route of the Yutu rover. a Geological context of the landing site. The landing site (white star) is located on the rim of the $\sim 450 \mathrm{~m}$ diameter Ziwei crater. The base image is obtained by the Lunar Reconnaissance Orbit (M102285549LE+RE; 1.66 m/pixel). b Image acquired by the descent camera (image ID: CE3_BMYK_LCAM-3006) on the Chang'e-3 lander shows numerous small craters within the landing site. The landing site is marked by the white star and the white line is the route of the Yutu rover. $\mathbf{c}$ Traverse route and speed of the Yutu rover from the navigation points N101 to N208 (black dots). The color bar is for the moving speed of the rover. Navigation points that are started with ' 1 ' represent the first lunar day, and ' 2 ' represents the second lunar day. The white star represents the position of lander 
points (Ling et al. 2015), and it is our target to resolve the $\mathrm{TiO}_{2}+\mathrm{FeO}$ content along the route of the Yutu rover.

Measurements for the permittivity of returned lunar regolith revealed that at radar frequencies larger than $10^{5}$ $\mathrm{Hz}$, the loss tangent of lunar regolith is primarily affected by the content of $\mathrm{TiO}_{2}+\mathrm{FeO}$, while the loss tangent is only weakly related to the bulk density (Strangway et al. 1977). On the other hand, the Apollo 17 mission performed both radar and seismic detections for the subsurface, and a sudden increase was observed in both the imaginary and real parts at the possible boundary between the regolith layer and the competent basalts, and this increase was interpreted to be caused by the larger bulk density of competent basalts (Strangway et al. 1977). The top $\sim 50 \mathrm{~ns}$ of the CE-3 LPR radargram is restricted within regolith-like materials (Xiao et al. 2015; Lai et al. 2016), so that the content of $\mathrm{TiO}_{2}+\mathrm{FeO}$ can be estimated using the loss tangent derived from the LPR data.

Loss tangent can be estimated in both the time-domain (i.e., a decay function of signal amplitude with time; Brzostowski and McMechan, 1992) and frequency-domain (i.e., a decrease function of centroid frequency with time; Brzostowski and McMechan, 1992; Turner and Siggins, 1994). Compared to the time-domain method, the advantage of the frequency-domain method is that the frequency shift of the reflected electromagnetic waves is not affected by reflection losses or far-field geometrical spreading (Liu et al. 1998). Difficulties raised by the amplitude decay of the reflected electromagnetic waves can be ignored using frequency-domain method.

The relationship between the dielectric losses and downshift of the centroid frequency (Quan and Harris, 1997) can be applied to estimate the loss tangent of the lunar regolith (Irving and Knight, 2003; Lauro et al. 2017). This method is also widely used to estimate the loss tangent of media both in seismic detections (e.g. Quan and Harris, 1997) and ground penetrating radar (Irving and Knight, 2003; Liu et al. 1998; Quan and Harris, 1997). When electromagnetic waves are propagating in loss materials such as lunar regolith, the centroid frequency of the received electromagnetic wave $\left(f_{r}\right)$ is lower than that transmitted $\left(f_{t}\right)$, a phenomena called wavelet dispersion, which is caused by dielectric losses (Turner and Siggins, 1994). To estimate the loss tangent using the frequency shift method, the LPR data are transformed to the frequency domain, and the centroid frequency and its time decay for each trace of the LPR data are calculated using the short-time Fourier transformation (STFT; Griffin and Lim, 1984). The decreasing rate of the centroid frequency of the received signal (i.e., downtrend slope) is computed using a linear least square fit (Irving and Knight, 2003). Loss tangent is

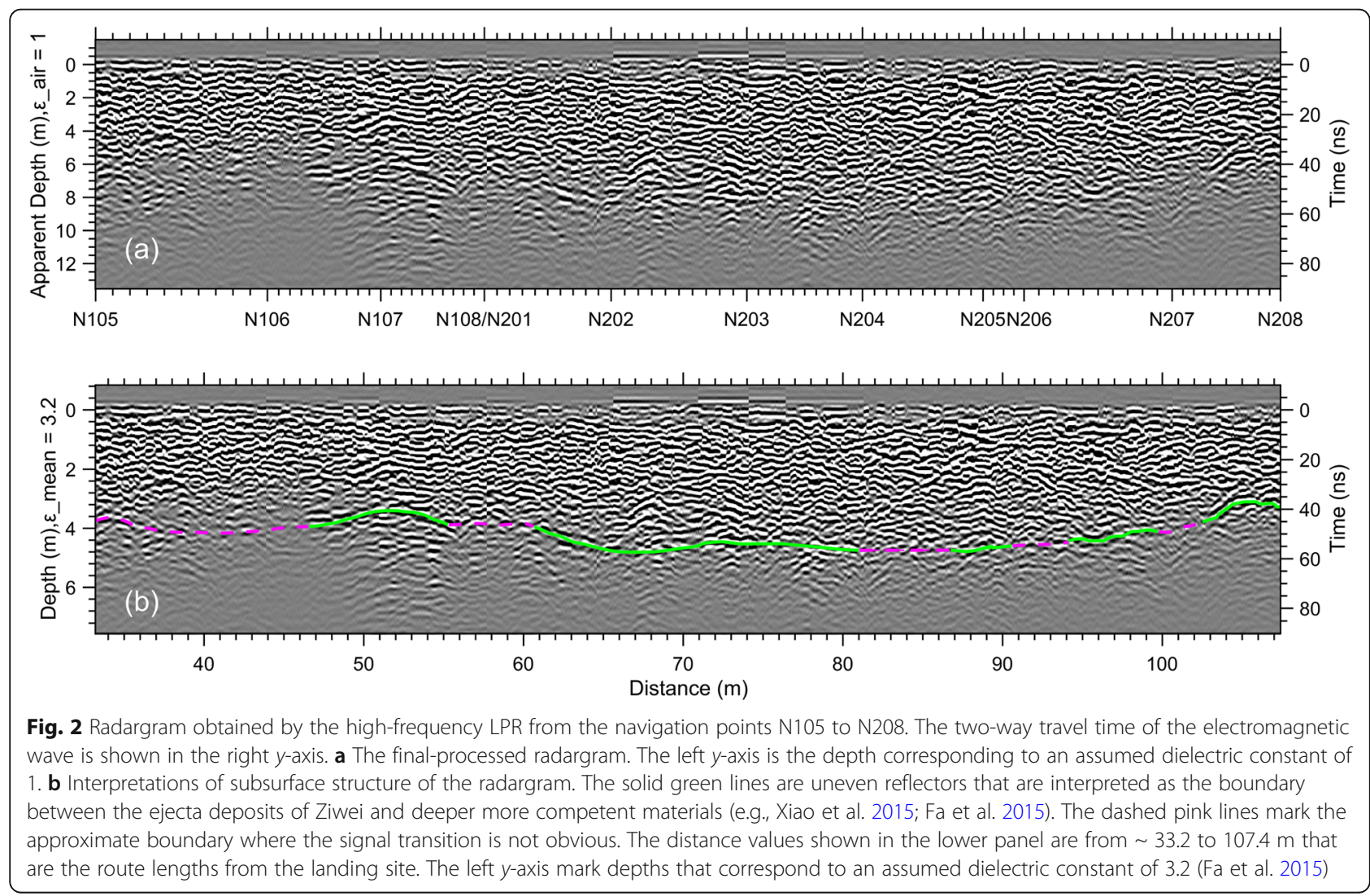


proportional to the downtrend slope (Quan and Harris, 1997; Irving and Knight, 2003).

The frequency drift of the received electromagnetic waves is calculated based on the assumption that for electromagnetic waves in radar wavelengths, the amplitudes of the transmitted signals $(x)$, those loss through medium ( $r)$, and those of the received signals $(y)$ follow a linear system (Liu et al. 1998; Quan and Harris, 1997). Therefore, at each time $(\tau)$, the amplitude of the transmitted and that of the received radar waves can be calculated via convolution based on the amplitude losses in the medium as shown in Fig. 3. In a linear signal system, the time-domain signal can be translated to a frequencydomain signal using the Fourier transformation (FFT; Rabiner and Gold, 1975), which can simplify the calculation procedure (Cooley et al. 1969). Equation (1) shows the linear system in the frequency domain (Fig. 3).

$$
Y(f)=X(f) \cdot R(f)
$$

where $f$ is frequency, $Y(f)$ is the frequency spectrum of the received signal. $X(f)$ is the frequency spectrum of the transmitted radar pulse. $R(f)$ is the propagation function of the radar pulse, which includes both attenuation and phase terms. The transmitted radar pulse of the high frequency LPR channel can be approximated as a plane wave (Irving and Knight, 2003), so that the frequency spectrum of the received signal $Y(f)$ at a given distance $d$ can be stated as Eq. (2).

$$
Y(f)=X(f) \cdot e^{-\alpha d} \cdot e^{-j \beta d}
$$

where $\alpha$ is the attenuation term, and $\beta$ is the phase term in the loss medium $R(f)$. The loss tangent $(\tan \delta)$ of the lossy medium is proportional to the attenuation function $(\alpha)$, and their relationship is expressed as Eq. (3). Constant $\tan \delta$ not depending on frequency is assumed in this study for simplicity.

$$
\alpha=\frac{\omega}{v_{p}}\left\{\frac{\left[\sqrt{1+\tan ^{2} \delta-1}\right]}{2}\right\}^{\frac{1}{2}}
$$

where $\omega=2 \pi f, f$ is the frequency of the radar pulse. $v_{p}$ is the velocity of the radar pulse in the medium. The loss tangent of typical lunar regolith is $~ 0.005$ (Strangway et al. 1977), so that the power of the loss tangent $\left(\tan ^{2} \delta\right)$ is far less than one. Applying the binomial approximation $\sqrt{1+\chi^{2}}-1 \approx \chi^{2} / 2$ to Eq. (3), we obtain

$$
\alpha(f)=\frac{\pi \tan \delta}{v_{p}} f
$$

The electromagnetic wave transmitted by the highfrequency LPR follows a constant Ricker function (Fang et al. 2014), which can also be approximated by the Gaussian function shown in Eq. (5) (Lauro et al. 2017):

$$
X(f)=\frac{2}{f_{0} \sqrt{\pi}} \exp \left[-\frac{4\left(f-f_{0}\right)^{2}}{f_{0}^{2}}\right]
$$

where $f_{O}$ is the dominant frequency of the transmitted radar pulse, which is also recognized as the peak frequency (Zhang et al. 2002). Substituting Eq. (4) and (5) into Eq. (2), the frequency spectrum of the received signal can be expressed as the Eq. (6). More detailed derivation process can be found in the supplementary information..

$$
\begin{aligned}
& Y(f)= \frac{2}{f_{0} \sqrt{\pi}} \exp \left\{-\frac{4\left[f-\left(f_{0}-\frac{\pi \tan \delta f_{0}^{2} d}{8 v_{p}}\right)\right]^{2}}{f_{0}^{2}}\right\} \\
& \cdot \exp \left[\left(\frac{\pi \tan \delta f_{0} d}{4 v_{p}}-2\right)^{2}-4\right] \\
& \cdot \exp (-j \beta d)
\end{aligned}
$$

The first exponential expression of Eq. (6) is the Gaussian format of the transmitted radar pulse. The second is the attenuation of the radar pulse, and the third is the phase of the radar pulse.

The centroid frequency of the received signal $\left(f_{r}\right)$ is shown in the Eq. (7) (Irving and Knight, 2003; Liu et al. 1998; Quan and Harris, 1997).

$$
f_{r}=\frac{\int_{0}^{\infty} f \cdot Y(f) d f}{\int_{0}^{\infty} Y(f) d f}
$$

The centroid frequency of transmitted radar wave $X(f)$ is $f_{t}$, which is expressed as Eq. (8) (Quan and Harris, 1997).

$$
f_{t}=\frac{\int_{0}^{\infty} f \cdot X(f) d f}{\int_{0}^{\infty} X(f) d f}=f_{0}
$$

Combining Eqs. (6), (7), and (8), the centroid frequency of the received signal $\left(f_{r}\right)$ is related with that of centroid frequency of the transmitted signal $\left(f_{t}\right)$ and the loss tangent (Quan and Harris, 1997), and expressed as

$$
f_{r}=f_{t}-\frac{\pi \tan \delta f_{t}^{2}}{8} \tau
$$

where $\tau=d / v_{p}$ is propagation time. Fitting the centroid frequency of the received signal $\left(f_{r}\right)$ and the propagation time $(\tau)$, we can estimate the loss tangent $(\tan \delta)$ of the electromagnetic wave and the centroid frequency of the transmitted signal $\left(f_{t}\right)$. Equation (10) shows the relationship between loss tangent $(\tan \delta)$ and the frequency drift $\operatorname{rate}\left(\Delta f_{r} / \Delta \tau\right)$. 


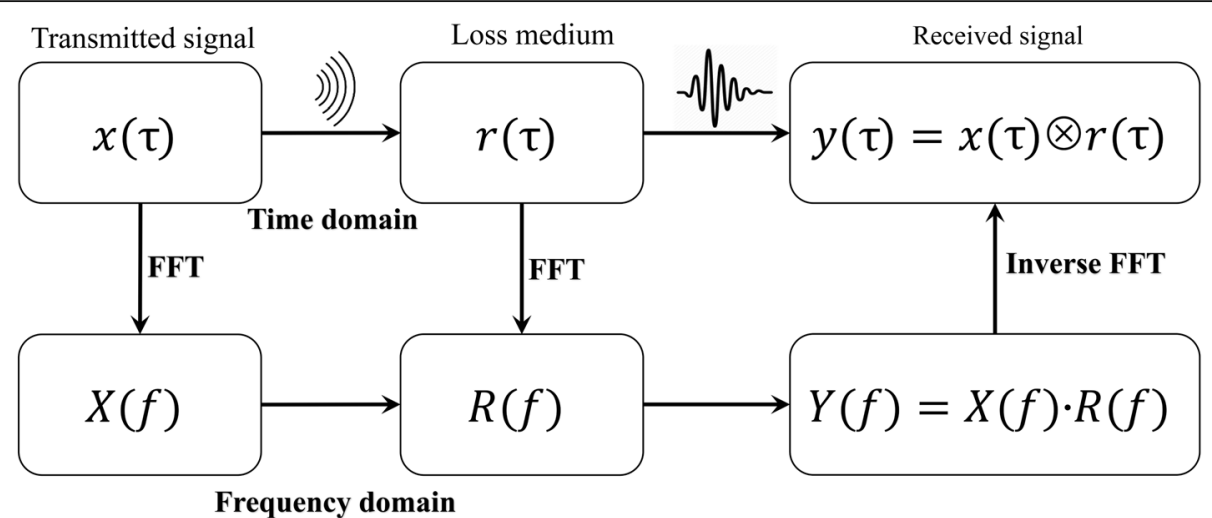

Fig. 3 The LPR data are treated as a linear signal system. Fourier transformation and Inverse Fourier transformation are used to transform the signal system from time domain to frequency domain (Rabiner and Gold, 1975). In the time domain, the received signal $y(\tau)$ is derived based on the convolution between the transmitted signal $x(\tau)$ and that of the loss medium $r(\tau)$. In the frequency domain, the amplitude spectrum of the received signal $Y(f)$ equals the product of the amplitude spectra of the transmitted waveform $X(f)$ and the loss medium $R(f)$

$$
\tan \delta=-8 \frac{\Delta f_{r} / \Delta \tau}{\pi f_{t}^{2}}
$$

In the calculation, STFT is applied to the first $\sim 50 \mathrm{~ns}$ of each A-scan to transform the received LPR signal from time-domain, $y(\tau)$, to frequency-domain, $Y(f)$. The $\sim 50 \mathrm{~ns}$ boundary (i.e., solid green and dashed pink curves; Fig. $2 \mathrm{~b}$ ) corresponds to the approximated depth of surface regolith along the rover route. The sampling frequency is set to 3.2 $\mathrm{GHz}$, which corresponds to the time sampling interval (0.3125 ns) of the high frequency LPR (Su et al. 2014). Each A-scan is divided into 8 equally sized segments (i.e., $\sim 6 \mathrm{~ns}$ each), and the overlapped time width is set to $50 \%$ of the segment width (i.e., 3 ns) to ensure reliable frequency resolution (Niethammer et al. 2000). A 3 ns Hamming window is applied for each segment of the A-scan for the STFT.

\section{Results}

\section{Estimate of loss tangent}

For each segment of the LPR data that were obtained between adjacent navigation points (Fig. 1), the frequency drift rate for the radar data is calculated. Figure 4 shows the centroid frequency of the received signals versus timedelays, which are fitted using linear equations. It is notable that $f_{r}$ here is derived from the data after reducing the system noise. Deriving $f_{r}$ from the raw data would cause substantial oscillation in the echo pattern. Table 1 shows the results and the related errors. In general, the loss tangent derived is $\sim 0.011-0.017$ (Table 1 ), and the average value is 0.014 . Referring to the relationship between loss tangent and penetration depth of the high-frequency LPR (Xing et al. 2017; Fig. 5a), our estimated loss tangent corresponds to penetrating depths of $\sim 12.5-15.7 \mathrm{~m}(\varepsilon=2.9)$, which is consistent with the actual penetrating depth of the high-frequency LPR (Xiao et al. 2015; Fa et al. 2015).

\section{Bulk $\mathrm{TiO}_{2}+\mathrm{FeO}$ content}

The average density of materials within the top $\sim 50 \mathrm{~ns}$ of LPR data is estimated to be $1.8 \mathrm{~g} / \mathrm{cm}^{3}$ (Fa et al. 2015), which is consistent with the density of the typical lunar regolith (Carrier et al. 1991). Therefore, applying the empirical relationship between the loss tangent and $\mathrm{TiO}_{2}+$ $\mathrm{FeO}$ content of lunar regolith samples (Eq. 11; Carrier et al. 1991), the bulk $\mathrm{TiO}_{2}+\mathrm{FeO}$ content of materials within the $\sim 50 \mathrm{~ns}$ of the radargram can be calculated.

$$
\tan \delta=10^{\left(0.030 \times\left(\% \mathrm{TiO}_{2}+\% \mathrm{FeO}\right)-2.676\right)}
$$

The $\mathrm{TiO}_{2}+\mathrm{FeO}$ content is estimated as 23-30 wt.\% (Table 1 ), and the average value is $\sim 27 \mathrm{wt} . \%$.

The estimated bulk concentration of $\mathrm{TiO}_{2}+\mathrm{FeO}$ exhibits a decreasing trend from the navigation points N105 to N202 (Fig. 5b). In general, navigation points further away from the rim of Ziwei (Fig. 1) exhibit a higher content of $\mathrm{TiO}_{2}+\mathrm{FeO}$ (Fig. 5b). However, the $\mathrm{TiO}_{2}+\mathrm{FeO}$ concentration at N107-N108 is distinctly less than the surroundings, suggesting that the composition of materials in this region is patchy in distribution. Assuming that the change of the bulk $\mathrm{TiO}_{2}+\mathrm{FeO}$ contents along the traverse route of Yutu is continuous outward, the $\mathrm{TiO}_{2}+\mathrm{FeO}$ contents along the route of Yutu are interpolated to derive a regional trend (Fig. 5c) using the natural neighbor method (Sibson, 1981).

\section{Discussion}

Reliability of the loss tangent estimated

In this study, the loss tangent of subsurface materials along the route of Yutu is estimated using the relationship between the centroid frequency of the received signals and the time delay. Table 1 shows that the centroid frequency of the LPR transmitter is $~ 495.1-510.4 \mathrm{MHz}$. The average value $(501.3 \mathrm{MHz})$ is identical with that of 

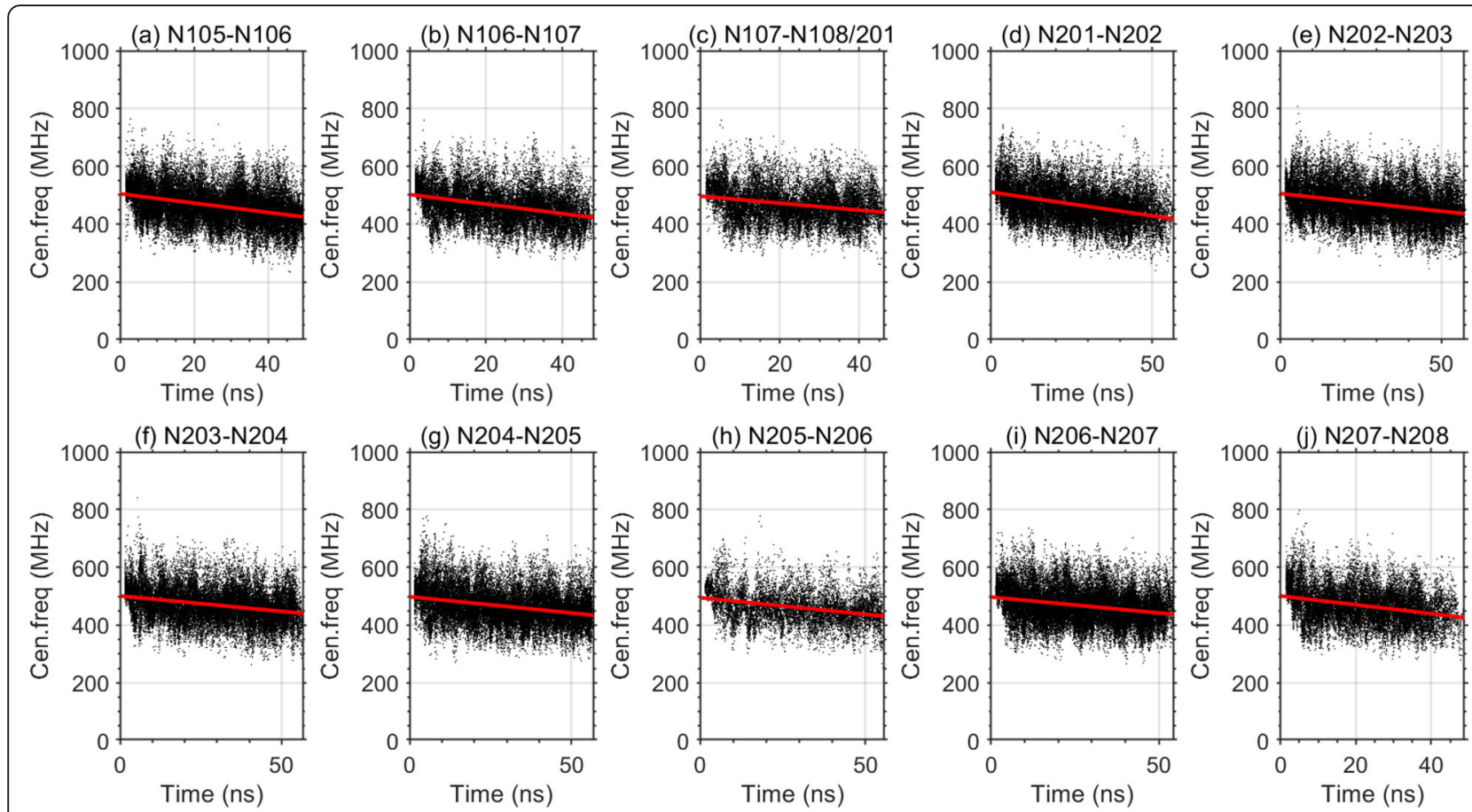

Fig. 4 The relationship between the centroid frequency of the received signal $\left(f_{r}\right)$ and the time-delay for each segment of the LPR data. The data segments $(\mathbf{a}-\mathbf{j})$ are divided according to the adjacent navigation points from N105 to N208. The red lines are the best-fit between $f_{r}$ and $\tau$ using the least square method. The slopes of the best-fit lines are the drift rate of $f_{r}$ with $\tau$

the designed centroid frequency $(500 \mathrm{MHz})$ of the high frequency LPR (Fang et al. 2014), suggesting that the base data used to estimate the loss tangent is reliable.

The top $\sim 50 \mathrm{~ns}$ of the CE-3 LPR data are restricted within the continuous ejecta deposits of the Ziwei crater (Xiao et al. 2015; Fa et al. 2015). The ejecta deposits are dominated by pre-impact regolith as evident by the geological context (Qiao et al. 2016). This is also consistent with the relative permittivity value of $\sim 2-5$ estimated using the LPR data (Fa et al. 2015; Lai et al. 2016; Feng et al. 2017), which is substantially less than that of typical lunar rocks (Carrier et al. 1991). The derived loss tangent values shown in Table 1 are within the range and toward the upper end of the measured loss tangent of returned regolith samples by the Apollo missions (0.00057 to 0.0232; Carrier et al. 1991). Typical lunar regolith samples exhibit loss tangent less than 0.01 , and this is also true for in situ measurements performed on the Moon. The Surface Electrical Properties Experiment carried out by the Apollo 17 mission has measured the permittivity and loss tangent of materials at the landing site, and the interpreted regolith layer ( $7 \mathrm{~m}$ thick) has a bulk loss tangent of 0.008 and a relative permittivity of 3.8 (Strangway et al. 1977). On the contrary, the loss tangent of the interpreted bedrocks at the Apollo 17 landing site is 0.035 and the relative permittivity is 7.7 (Strangway et al. 1977). It is notable that the value of loss tangent cannot be used to determine whether or not

Table 1 Parameters estimation for each navigational points. The centroid frequency of the received radar signal, the drift slope of the centroid frequency, the derived loss tangent, and the derived $\mathrm{TiO}_{2}+\mathrm{FeO}$ contents at different segments of the LPR data. The uncertainties for all the estimated values are the $95 \%$ confidence bounds. The navigation points are shown in Figs. 1c and $5 \mathrm{~b}$

\begin{tabular}{|c|c|c|c|c|}
\hline $\begin{array}{l}\mathrm{LPR} \\
\text { segments }\end{array}$ & $\begin{array}{l}\text { Centroid frequency } \\
\left(f_{t}, \mathrm{MHz}\right)\end{array}$ & $\begin{array}{l}\text { Frequency drift rate } \\
\left(\Delta f_{r}, \mathrm{MHz} / \mathrm{ns}\right)\end{array}$ & $\begin{array}{l}\text { Loss tangent } \\
(\tan \delta)\end{array}$ & $\mathrm{TiO}_{2}+\mathrm{FeO}($ wt.\%) \\
\hline $\begin{array}{l}\text { N105-N106 } \\
\text { N106-N107 } \\
\text { N107-N108 } \\
\text { N108-N202 } \\
\text { N202-N203 } \\
\text { N203-N204 } \\
\text { N204-N205 } \\
\text { N205-N206 } \\
\text { N206-N207 } \\
\text { N207-N208 }\end{array}$ & $\begin{array}{l}504.75 \pm 1.22 \\
502.10 \pm 1.58 \\
496.52 \pm 1.70 \\
510.37 \pm 1.40 \\
505.30 \pm 1.31 \\
501.44 \pm 1.41 \\
498.57 \pm 1.38 \\
495.11 \pm 2.28 \\
497.40 \pm 1.30 \\
501.24 \pm 1.74\end{array}$ & $\begin{array}{l}-1.623 \pm 0.043 \\
-1.661 \pm 0.059 \\
-1.217 \pm 0.066 \\
-1.653 \pm 0.047 \\
-1.219 \pm 0.040 \\
-1.079 \pm 0.044 \\
-1.128 \pm 0.042 \\
-1.152 \pm 0.071 \\
-1.107 \pm 0.043 \\
-1.536 \pm 0.070\end{array}$ & $\begin{array}{l}0.0162 \pm 0.0004 \\
0.0168 \pm 0.0005 \\
0.0126 \pm 0.0006 \\
0.0162 \pm 0.0004 \\
0.0122 \pm 0.0003 \\
0.0109 \pm 0.0004 \\
0.0116 \pm 0.0004 \\
0.0120 \pm 0.0006 \\
0.0114 \pm 0.0004 \\
0.0156 \pm 0.0006\end{array}$ & $\begin{array}{l}29.54 \pm 0.33 \\
30.02 \pm 0.44 \\
22.58 \pm 0.70 \\
29.48 \pm 0.35 \\
25.36 \pm 0.41 \\
23.82 \pm 0.52 \\
24.63 \pm 0.47 \\
25.13 \pm 0.77 \\
24.42 \pm 0.50 \\
28.94 \pm 0.58\end{array}$ \\
\hline
\end{tabular}




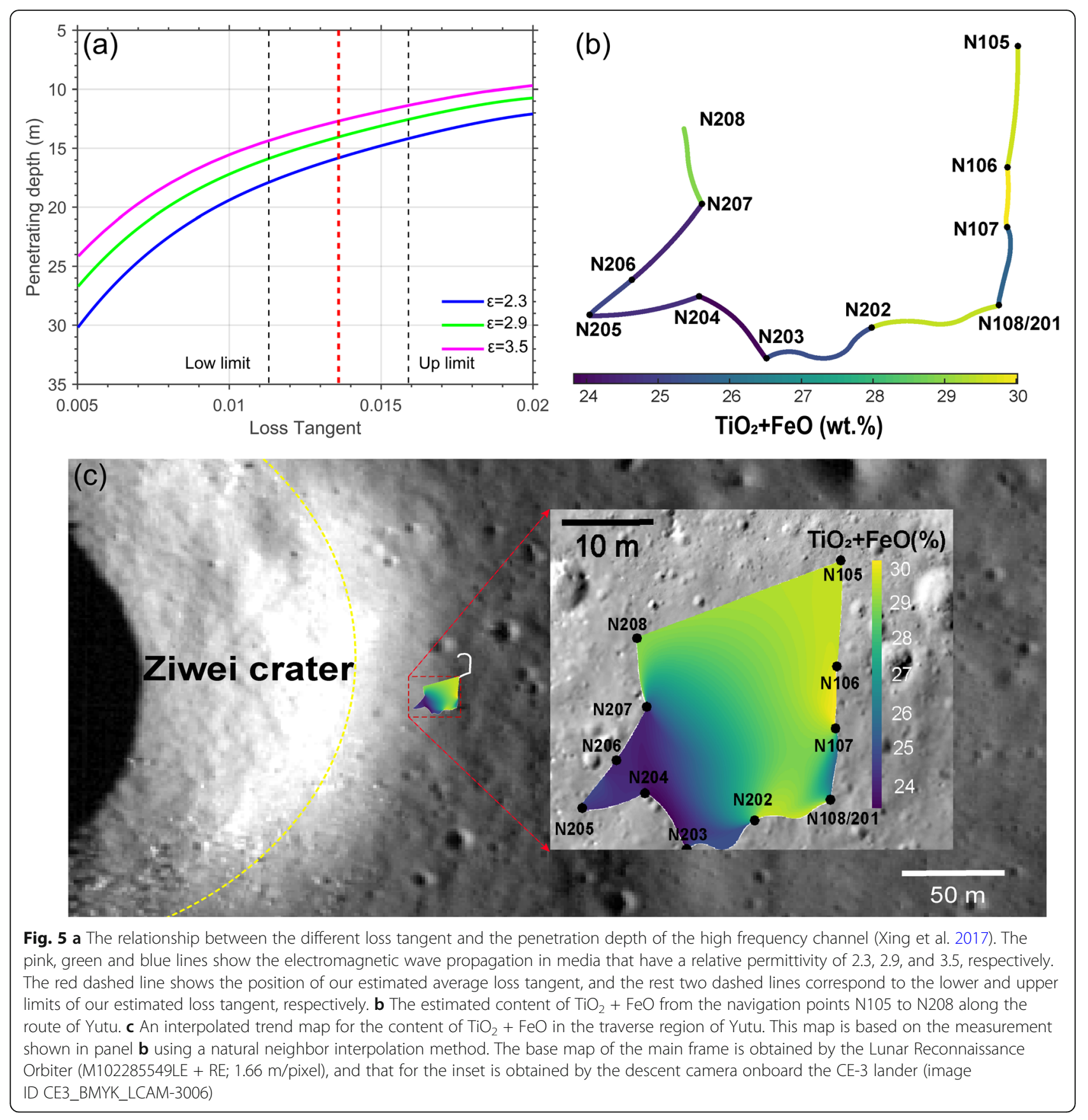

the medium is porous regolith or competent bedrock. The Lunar Radar Sounder (operating frequency of $5 \mathrm{MHz}$ ) onboard the Kaguya orbiter (SELENE) estimated that the loss tangent for the $\sim 200 \mathrm{~m}$ thick mare units at the Oceanus Procellarum is $\sim 0.001-0.01$, but the estimated relative permittivity is $\sim 5.76-8.08$ (Ono et al. 2009).

\section{Reliability of the $\mathrm{TiO}_{2}+\mathrm{FeO}$ contents estimated}

The $\mathrm{TiO}_{2}+\mathrm{FeO}$ contents derived from the loss tangent are the average values for materials within the top $\sim 50 \mathrm{~ns}$ of the LPR data. This value is consistent with various orbital and in situ observations for this region. The Gamma Ray Spectroscopy onboard the Lunar Prospector find that the $\mathrm{TiO}_{2}+\mathrm{FeO}$ content on the mare surface where the CE-3 landed is $25.2 \mathrm{wt} . \%$ (Prettyman et al. 2006). The ultraviolet-visible spectrometer onboard the Clementine mission found that the $\mathrm{TiO}_{2}+\mathrm{FeO}$ near the CE-3 landing site is $24-26$ wt.\% (with a FeO content of 19 wt.\% and a $\mathrm{TiO}_{2}$ content of 5-7 wt.\%; Ling et al. 2015). Likewise, the $\mathrm{FeO}$ and $\mathrm{TiO}_{2}$ content estimated by the Multispectral Imager onboard the Kaguya mission (Fig. 6a, b) are also consistent with the measurements 

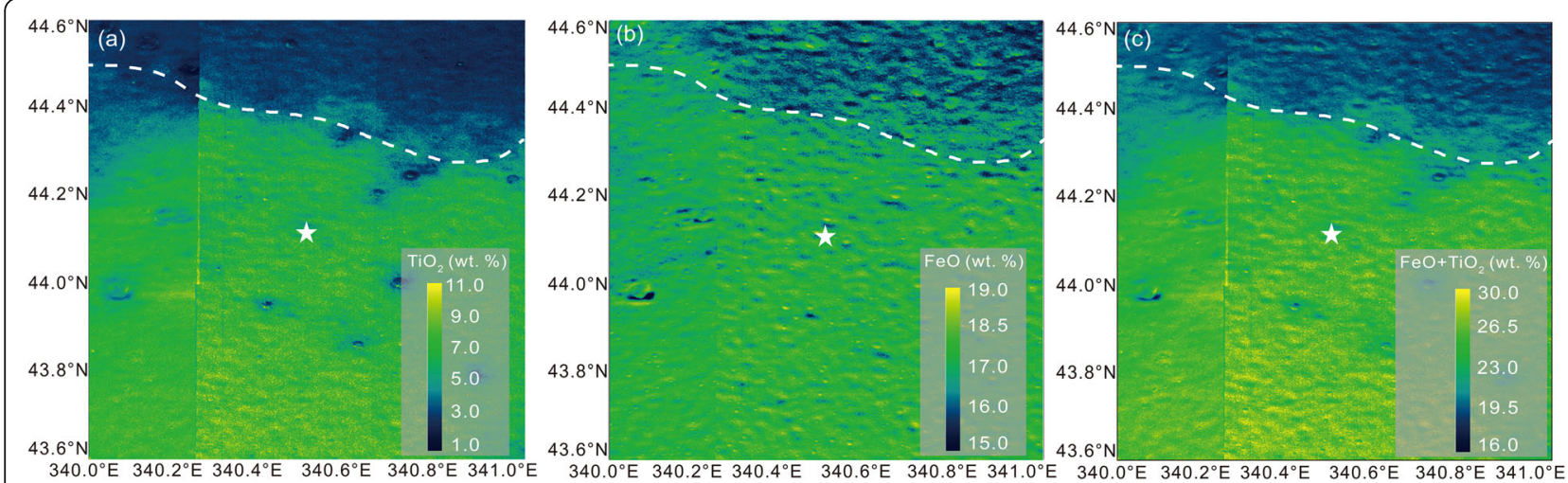

Fig. 6 The $\mathrm{TiO}_{2}(\mathbf{a}), \mathrm{FeO}(\mathbf{b})$, and their combined (c) contents at the CE-3 landing site (white stars). The data are derived from the Multispectral Imager data, and the maps are revised from Zhao et al. (2014). The white dashed line is the boundary between the Imbrian- (north) and Eratosthenian-aged mare units (down). North is up in all the panels

done here (Fig. 6c and Table 1), which are basically consistent those estimated by the Clementine mission (Zhao et al. 2014). Furthermore, in situ measurements performed by the CE-3 APXS found that the $\mathrm{TiO}_{2}+\mathrm{FeO}$ content is $\sim 27.8$ wt.\% (Ling et al. 2015). Therefore, the estimated $\mathrm{TiO}_{2}+\mathrm{FeO}$ content are in line with previous studies.

The higher content of $\mathrm{TiO}_{2}+\mathrm{FeO}$ compared to the global average of mare surfaces (12\%; Lucey et al. 1995) explains the larger than average loss tangent derived in Table 1. The measured loss tangent of returned lunar regolith samples exhibit a large range (from less than 0.001 to 0.03 ), indicating that the loss tangent of lunar regolith is weakly dependent on the density or relative permittivity and the dominant factor is the content of $\mathrm{TiO}_{2}+\mathrm{FeO}$ (Olhoeft et al. 1975; Strangway et al. 1977). Regolith samples that have $>0.01$ loss tangent uniformly feature a high content of $\mathrm{TiO}_{2}+\mathrm{FeO}$ (Table 1). Therefore, while the CE3 landing region has the highest content of $\mathrm{TiO}_{2}+\mathrm{FeO}$ among lunar mare surfaces (Zhao et al. 2014), the regolith layer, which is the dominant materials within the ejecta deposits of Ziwei, also features the largest loss tangent.

\section{Indications to regional geology}

Along the route of Yutu, places further away from the rim of Ziwei generally exhibit a slightly larger $\mathrm{TiO}_{2}+\mathrm{FeO}$ content of $~ 30$ wt.\%, e.g., navigation points N105-N107 (Fig. 5b). This content is comparable with that of the Eratosthenian-aged mare surface around the landing site (white star in Fig. 6c). However, places closer to the rim of Ziwei have a content of $\mathrm{TiO}_{2}+\mathrm{FeO} \sim 24$ wt.\% (e.g., navigation points N203-N207; Fig. 5b), which is closer to the Late Imbrian-aged mare basalts to the north (Fig. 6c). This comparison indicates that the generally lower content of $\mathrm{TiO}_{2}+$ $\mathrm{FeO}$ closer to the rim of Ziwei may be caused by the impact excavation of deeper Imbrian-aged materials, as the Eratosthenian-aged basalts have been penetrated through by Ziwei. Also, the route between navigation points N107-
N108 features a less content of $\mathrm{TiO}_{2}+\mathrm{FeO}$ compared to the surrounding area, indicating that the excavated low $\mathrm{TiO}_{2}+\mathrm{FeO}$ materials follow a patchy distribution. Since the relative permittivity of materials within the top $\sim 50 \mathrm{~ns}$ of the radargram are close to that of lunar regolith (e.g., Fa et al. 2015), the excavated Imbrian-aged materials are most likely from the paleo-regolith that was developed between the eruptions of the Erathothenian- and Imbrian-aged mare basalts. This interpretation is consistent with the geological interpretation of the LPR data (Xiao et al. 2015). Furthermore, most of the continuous ejecta deposits of the Ziwei crater exhibit a high content of $\mathrm{TiO}_{2}+\mathrm{FeO}$ (Fig. 6c), suggesting that most of ejecta is from shallow part of Ziwei crater site and Imbrium-agedpaleo-regolith with low $\mathrm{TiO}_{2}$ $+\mathrm{FeO}$ low contents from deep part is minor and limited to crater rims and restricted locations (e.g., N107-N108; Fig. 5c) because Ziwei crater is small (Qiao et al. 2016).

The $\mathrm{TiO}_{2}+\mathrm{FeO}$ content derived here bridges the resolution gap between in situ and orbital observations, attesting the advantage of ground penetration radar in estimating the bulk composition of regolith materials. Compared to compositional data obtained by orbital observations (e.g., the maps shown in Fig. 6 represent the highest resolution maps obtained from orbit), the large variations of the $\mathrm{TiO}_{2}+\mathrm{FeO}$ content along the route of Yutu (Fig. 5b, c) is not expected (Fig. 6c), appealing for more careful interpretations of compositional data obtained from orbit.

\section{Conclusion}

The high-frequency radar data obtained by the lunar penetration radar onboard the Yutu rover, Chang'e-3 is used to derive the content of $\mathrm{TiO}_{2}+\mathrm{FeO}$ for the shallow materials along the route of Yutu. The frequency-shift method is used for the top $\sim 50 \mathrm{~ns}$ radar data to evaluate the drift rates of the received radar signals, which are related to the loss tangent of the bulk materials. The estimated loss tangent is $\sim 0.011-0.017$, which is consistent with, but slightly 
larger than the measured results for returned lunar regolith. The larger than average loss tangent for the bulk material along the route of Yutu is mainly caused by of the high content of $\mathrm{TiO}_{2}+\mathrm{FeO}(\sim 23-30$ wt.\%), which is consistent with both orbital and in situ observations (Prettyman et al. 2006; Ling et al. 2015; Zhang et al. 2015). The resolved $\mathrm{TiO}_{2}+\mathrm{FeO}$ contents exhibit a lower value towards the rim of Ziwei, which is caused by the excavation of the Late-Imbrian-aged paleo-regolith. This study shows that ground penetration radar can be applied as a useful supplementary tool to investigate the bulk composition of lunar regolith, as both the spatial resolution and detection depth are better than orbital observations.

\section{Supplementary information}

Supplementary information accompanies this paper at https://doi.org/10. 1186/s40645-020-00340-4.

Additional file 1. Supplementary material.

\section{Abbreviations}

LPR: Lunar penetrating radar; CE-3: Chang'e-3; SELENE: Selenological and Engineering Explorer; APXS: Active particle-induced X-ray spectrometer; FFT: Fast Fourier transformation; STFT: Short time Fourier transformation

\section{Acknowledgements}

We thank Dr. Sebastian Emanuel Lauro and Dr. Federico Di Paolo for helpful discussion. Comments and suggestions provided by two anonymos reviewers significantly helped clarifying and improving the manuscript.

\section{Authors' informations \\ Dr. Chunyu Ding is an associate researcher at Sun Yat-sen University. He received a Ph.D of Astronomy from the National Astronomical Observatories, Chinese Academy of Sciences in 2017. Dr. Zhiyong Xiao is an associate professor at the Planetary Environmental and Astrobiological Research Laboratory, Sun Yat-sen University. Dr Jiannan Zhao is a postdoc at China University of Geosciences (Wuhan). Dr. Yan Su is the PI of data acquisition subsystem at the Ground Application System of Lunar Exploration, National Astronomical Observatories, Chinese Academy of Sciences. Dr. Jun Cui is the director of Planetary Environmental and Astrobiological Research Laboratory, Sun Yat-sen University.}

\section{Authors' contributions}

C.D. and Z.X. proposed the topic, conceived and designed the study, and analyzed the data. Y.S, J.Z., and J.C. collaborated with the corresponding author in the construction of manuscript. All authors read and approved the final manuscript.

\section{Funding}

The authors are supported by the B-type Strategic Priority Program of the Chinese Academy of Sciences (XDB41000000), the National Natural Science Foundation of China (41773063, 41525015, and 41830214), the Science and Technology Development Fund of Macau (0042/2018/A2), the Pre-research Project on Civil Aerospace Technologies (D020101, D020202) of CNSA, and the Opening Fund of the Key Laboratory of Lunar and Deep Space Exploration, Chinese Academy of Sciences (Idse201702; Idse201908).

\section{Availability of data and materials}

Source codes and data used in this study open to public access (https://doi. org/10.5281/zenodo.3884522). The LPR data are also available at the Ground Application System of Lunar Exploration, National Astronomical Observatories, Chinese Academy of Sciences (http://moon.bao.ac.cn).

\section{Competing interests}

The authors declare that they have no competing interests.

\section{Author details}

${ }^{1}$ Planetary Environmental and Astrobiological Research Laboratory, School of Atmospheric Sciences, Sun Yat-sen University, Zhuhai, China. ${ }^{2}$ Key Laboratory of Lunar and Deep Space Exploration, National Astronomical Observatories, Chinese Academy of Sciences, Beijing, China. ${ }^{3}$ State Key Laboratory of Lunar and Planetary Sciences, Space Science Institute, Macau University of Science and Technology, Macau, China. ${ }^{4}$ Chinese Academy of Sciences, Center for Excellence in Comparative Planetology, Hefei, China. ${ }^{5}$ University of Chinese Academy of Sciences, Beijing, China. ${ }^{6}$ Planetary Science Institute, School of Earth Sciences, China University of Geosciences (Wuhan), Wuhan, China.

Received: 28 October 2019 Accepted: 22 May 2020

Published online: 27 July 2020

\section{References}

Brzostowski MA, McMechan GA (1992) 3-D tomographic imaging of near-surface seismic velocity and attenuation. Geophysics 57(3):396-403.

Campbell BA, Hawke BR, Thompson TW (1997) Regolith composition and structure in the lunar maria: Results of long-wavelength radar studies. Journal of Geophysical Research: Planets 102(E8):19307-320.

Carrier D, Olhoeft R, Mendell W (1991) Physical properties of the lunar surface. In: Heiken GH, Vaniman DT, French BM(ed) Lunar source book-A user's guide to the moon.Cambridge University Press, Cambridge, pp 475-594.

Cooley W, Lewis W, Welch D (1969) The Fast Fourier Transform and Its Applications. IEEE Transactions on Education 12:27-34.

Fa W, Zhu M-H, Liu T, Plescia JB (2015) Regolith stratigraphy at the Chang'E-3 landing site as seen by lunar penetrating radar. Geophysical Research Letters 42(23):10,179-10,187.

Fang G, Zhou B, Ji Y, Zhang Q, Shen S, Li Y, Guang H, Tang C, Gao Y, Lu W, Ye S, Han H, Zheng J, Wang S (2014) Lunar Penetrating Radar onboard the Chang'e-3 mission. Research in Astronomy and Astrophysics 14(12):1607-22.

Feng J, Su Y, Ding C, Xing S, Dai S, Zou Y (2017) Dielectric properties estimation of the lunar regolith at CE-3 landing site using lunar penetrating radar data. Icarus 284:424-30.

Griffin D, Lim J (1984) Signal estimation from modified short-time Fourier transform. IEEE Transactions on Acoustics, Speech, and Signal Processing 32:236-43.

Huang Y, Minton D, Hirabayashi M, Elliott R, Richardson E, Fassett I, Zellner B (2017) Heterogeneous impact transport on the Moon. Journal of Geophysical Research: Planets 122(6):1158-80.

Irving D, Knight J (2003) Removal of wavelet dispersion from ground-penetrating radar data. Geophysics 68:960-70.

Lai J, Xu Y, Zhang X, Tang Z (2016) Structural analysis of lunar subsurface with Chang'E-3 lunar penetrating radar. Planetary and Space Science 120:96-102.

Lauro SE, Mattei E, Cosciotti B, Di Paolo F, Arcone SA, Viccaro M, Pettinelli E (2017) Electromagnetic signal penetration in a planetary soil simulant: Estimated attenuation rates using GPR and TDR in volcanic deposits on Mount Etna. Journal of Geophysical Research: Planets 122(7):1392-1404.

L3ing Z, Jolliff BL, Wang A, Li C, Liu J, Zhang J et al (2015) Correlated compositional and mineralogical investigations at the Chang'e-3 landing site. Nature Communications 6(1):8880.

Liu L, Lane JW, Quan Y (1998) Radar attenuation tomography using the centroid frequency downshift method. Journal of Applied Geophysics 40:105-16.

Lucey PG, Taylor GJ, Malaret E (1995) Abundance and Distribution of Iron on the Moon. Science. 268(5214):1150-53.

Niethammer M, Jacobs L, Qu J, Jarzynski J (2000) Time-frequency representation of Lamb waves using the reassigned spectrogram. The Journal of the Acoustical Society of America 107(5):L19-L24.

Olhoeft GR, Strangway DW (1975) Dielectric properties of the first 100 meters of the Moon. Earth and Planetary Science Letters 24(3):394-404.

Ono T, Kumamoto A, Nakagawa H, Yamaguchi Y, Oshigami S, Yamaji A, Kobayashi T, Kasahara Y, Oya H (2009) Lunar Radar Sounder Observations of Subsurface Layers Under the Nearside Maria of the Moon. Science 323(5916):909-12.

Pieters CM, Taylor LA, Noble SK, Keller LP, Hapke B, Morris RV, Allen CC, McKAY DS, Wentworth S (2000) Space weathering on airless bodies: Resolving a mystery with lunar samples. Meteoritics \& Planetary Science 35(5):1101-07.

Pommerol A, Kofman W, Audouard J, Grima C, Beck P, Mouginot J, Herique A, Kumamoto A, Kobayashi T, Ono T (2010) Detectability of subsurface interfaces in lunar maria by the LRS/SELENE sounding radar: Influence of mineralogical composition. Geophysical Research Letters 37:L03201.

Prettyman TH, Hagerty JJ, Elphic RC, Feldman WC, Lawrence DJ, McKinney GW, Vaniman DT (2006) Elemental composition of the lunar surface: Analysis of 
gamma ray spectroscopy data from Lunar Prospector. Journal of Geophysical Research: Planets 111:E12007.

Qiao L, Xiao ZY, Zhao JN, Xiao L (2016) Subsurface structures at the Chang'e-3 landing site: Interpretations from orbital and in-situ imagery data. Journal of Earth Science 27:707-15.

Quan Y, Harris JM (1997) Seismic attenuation tomography using the frequency shift method. Geophysics 62:895-905.

Rabiner LR, Gold B (1975) Theory and application of digital signal processing. Prentice-Hall, New Jersey.

Schaber GG, Thompson TW, Zisk SH (1975) Lava flows in mare imbrium: An evaluation of anomalously low earth-based radar reflectivity. The Moon 13: 395-423.

Sibson R (1981) A brief description of natural neighbor interpolation. In: Interpolating Multivariate Data. John Wiley, New York, pp 21-36.

Strangway D, Pearce G, Olhoeft G (1977) Magnetic and dielectric properties of lunar samples. In: The Soviet-American Conference on Cosmochmistry of the Moon and Planets, NASA Special Publication-370, Washington, pp 417-33.

Su Y, Fang G, Feng J, Xing S, Ji Y, Zhou B et al (2014) Data processing and initial results of Chang'e-3 lunar penetrating radar. Research in Astronomy and Astrophysics 14(12):1623-32.

Turner G, Siggins AF (1994) Constant Q attenuation of subsurface radar pulses. Geophysics 59:1192-1200.

Wu Y, Hapke B (2018) Spectroscopic observations of the Moon at the lunar surface. Earth and Planetary Science Letters 484:145-53.

Xiao L, Zhu P, Fang G, Xiao Z, Zou Y, Zhao J et al (2015) A young multilayered terrane of the northern Mare Imbrium revealed by Chang'E-3 mission. Science 347(6227):1226-29.

Xing S, Su Y, Feng J, Dai S, Xiao Y, Ding C, Li C (2017) The penetrating depth analysis of Lunar Penetrating Radar onboard Chang'e-3 rover. Research in Astronomy and Astrophysics 17(5):046.

Zhang C, Ulrych TJ (2002) Estimation of quality factors from CMP records. Geophysics 67(5):1542-47.

Zhang J, Yang W, Hu S, Lin Y, Fang G, Li C et al (2015) Volcanic history of the Imbrium basin: A close-up view from the lunar rover Yutu. Proceedings of the National Academy of Sciences 112(17):5342-47.

Zhang L, Zeng Z, Li J, Huang L, Huo, Hijun, Zhang J, Huai N (2019) A story of regolith told by Lunar Penetrating Radar. Icarus 321:148-60.

Zhao J, Huang J, Qiao L, Xiao Z, Huang Q, Wang J et al (2014) Geologic characteristics of the Chang'E-3 exploration region. Science China Physics, Mechanics and Astronomy 57(3):569-76.

\section{Publisher's Note}

Springer Nature remains neutral with regard to jurisdictional claims in published maps and institutional affiliations.

\section{Submit your manuscript to a SpringerOpen ${ }^{\circ}$ journal and benefit from:}

- Convenient online submission

- Rigorous peer review

- Open access: articles freely available online

- High visibility within the field

- Retaining the copyright to your article

Submit your next manuscript at $\boldsymbol{\nabla}$ springeropen.com 